\title{
THE SPECTRA OF COMETS 1950b (MINKOWSKI) AND 1951a (PAJDUSAKOVA)*
}

\author{
P. Swings and Thornton Page \\ University of Liége, Yerkes, and McDonald Observatories \\ Received August 4, 1951
}

\begin{abstract}
The spectrum of Comet 1950b at heliocentric distance 2.65 A.U. shows a strong solar continuum concentrated in the nucleus and $C N$ emission over a coma forty times larger. Comet 1951a at heliocentric distance $0.91 \mathrm{~A}$.U. is very unusual in showing a strong nuclear continuum and extremely weak $C N$ emission over a coma only eight times larger than the nucleus. The former seems normal for a comet distance from the sun; the latter is probably a "fresh" comet, newly arrived near the sun from the swarm of cometary material postulated by Oort at the outer confines of the solar system.
\end{abstract}

\section{INTRODUCTION}

Since 1940 a program of spectroscopic observations of comets has been carried on at the McDonald Observatory, with the aim of increasing our knowledge of the physical, chemical, and dynamical processes at play in comets. ${ }^{1,2,3,4}$ It was expected that new observational data would help in erecting a satisfactory theory of the origin and development of comets, a matter of importance in itself and in relation to theories of the origin and history of the solar system. The McDonald observers have tried to gather information on as many comets as possible. Special attention was devoted to the discovery of new cometary molecules $\left(\mathrm{OH}, \mathrm{NH}, \mathrm{CH}^{+}, \mathrm{CO}_{2}^{+}, \mathrm{NH}_{2}\right.$, and probably $\mathrm{OH}^{+}$were found), the establishment of the excitation mechanism (pure resonance fluorescence due to solar radiation), the spectroscopic differences between individual comets (e.g., in the intensity ratio of $O H$ and $N H$ ), the behavior of band intensities with distance to the sun, and the distribution of molecules within the coma and tail. Various laboratory investigations have been undertaken in conjunction with these cometary observations.

From recent theoretical investigations by Oort, van Woerkom, and Schmidt ${ }^{5}$ it appears that comets come from a swarm of material at the outer confines of the solar system. This swarm is definitely a part of the solar system, although it extends to distances of the order of several thousand times the distance of the farthest planet. It is through stellar perturbations of this swarm that "fresh" comets continue to come into the central parts of the solar system. The observational material permitting a spectroscopic comparison between "fresh," or new comets, and old ones, which have passed near the sun previously, is still rather scanty. This is due partly to the fact that sufficiently accurate data on the original orbits described before the comets came into the region of the planets are available only for comets observed before 1940. It is highly desirable that similar orbit calculations be performed for comets which have been well covered spectroscopically in recent years.

* Contributions from the McDonald Observatory, University of Texas, No. 206.

${ }^{1}$ Swings, Elvey, and Babcock, Ap. J., 94, 320, 1941.

${ }^{2}$ Swings and Page, $A p . J .$, 108, 526, 1948.

${ }^{3}$ Jose and Swings, $A$ p. J., 111, 41, 1950.

${ }_{4}^{4}$ Swings and Page, $A p . J ., 111,530,1950$.

${ }^{5}$ J. H. Oort, B.A.N., 11, 91, 1950; van Woerkom, B.A.N., 10, 445, 1948; M. Schmidt, B.A.N., 11, 253 , 1951; J. H. Oort and M. Schmidt, B.A.N., 11, 259, 1951; J. H. Oort, Observatory, 71, 129, 1951 (Halley Lecture), 
According to Oort and Schmidt, ${ }^{6}$ these spectroscopic data, scanty as they are, seem to indicate that comets showing an unusually strong continuous spectrum, not connected with outbursts or division, are new comets. In other words, the amount of dust relative to the observable gas appears to be larger in new comets. The photometric observations also indicate that the brightness of the new comets increases more slowly with diminishing heliocentric distance $r$ than in the case of older comets. The development of a comet's head would be due to at least two different processes: one causing the expulsion of dust and meteorites (process 1), the other giving rise to gases and hence to the usual emission bands (process 2). These processes vary differently with $r$. At large heliocentric distances, process 1 prevails, while process 2 dominates for distances below about 3 A.U. "The relative intensity of the two processes appears to vary considerably with the 'age' of the comet. In new comets process 1 is relatively much stronger, so that the continuous spectrum prevails even down to $r=1$, while the emission only begins to exceed it for still smaller $r .{ }^{\prime \prime 6}$ A theoretical treatment along the general lines of Levin's discussion ${ }^{7}$ indicates that the new comets deteriorate markedly during their first, or first few, perihelion passages. The complex physical mechanisms causing the production of dust and gas are still mostly unknown; their investigation by A. Delsemme and one of the authors (Swings) is under way.

In the coma of all comets-and especially so in new comets - the continuous spectrum prevails at large heliocentric distances. The few available observations indicate that at very large $r$ the spectra of comets are essentially continuous. This is the case for Comet Schwassmann-Wachmann I at $r=5.5$ after outbursts. ${ }^{8}$ Similarly, Halley's Comet at $r=2.8$ showed only a continuous spectrum ${ }^{9}$ with suspected $C N$ and possibly $C_{2}$. In Comet 1948l, at $r=2.21$, only the $C N$ and $\lambda 4050$ emissions remained conspicuous on a strong solar spectrum.

The data reported here on two recent comets, 1950b (Minkowski) and 1951a (Pajdusakova), have a direct bearing on Oort and Schmidt's theoretical discussions. The first comet, observed at $r=2.63$, provides data on comet spectra at fairly large heliocentric distances; moreover, it had the unusually large perihelion distance, $q=2.59$ A.U. The latter comet, observed at $r=0.92$, reveals an intense continuous spectrum, despite the small heliocentric distance and the small perihelion distance, $q=0.72$.

\section{THE OBSERVATIONAL MATERIAL}

Six spectra have been obtained with the B spectrograph and one with the quartz Cassegrain spectron raph attached to the 82-inch reflector of the McDonald Observatory. The instruments and procedure have been described previously. ${ }^{4}$ Detailed data are given in Table 1.

\section{THE SPECTRUM OF COMET 1950b (MINKOWSKI)}

At heliocentric distances between 2.62 and 2.66 A.U. the $(0,0)$ band of $C N$ is of fair intensity, while there is no trace of $C_{2}$. The solar spectrum is so intense in the central parts of the coma that no definite decision can be taken as to a possible weak emission near $\lambda$ 4050; at any rate, if any $\lambda 4050$ emission is present in the central regions, it must be much weaker than $C N$. On the spectrogram at $r=2.64$ there may be a trace of $\lambda 4050$ emission extending faintly on one side of the nuclear region. In Comet 19481 at $r=2.21, \lambda 4050$ was still conspicuous, ${ }^{3}$ being about as strong as the $R$ branch of $C N$ $(0,0)$ near the nucleus.

${ }^{6}$ Op. cit., p. 267.

${ }^{7}$ A.J.U.S.S.R., 20, 48, 1943; see also B. Voron tsov-Velyaminov, Ap. J., 104, 232, 1946; M. Minnaert, Proc. Kon. Ned. Akad. Wetensch., 50, 833, 1947; F. L. Whipple, Ap. J., 111, 385, 1950.

${ }^{8}$ N. U. Mayall, Pub. A.S.P., 53, 340, 1941; R. Minkowski, private communication.

${ }^{9}$ N. T. Bobrovnikoff, Pub. Lick Obs., 17, 447, 1931. 
The ultraviolet spectrogram of Comet $1950 \mathrm{~b}$ at $r=2.66$ reveals no trace of $O H$ or $\mathrm{NH}$, although $\mathrm{CN}$ appears fairly strongly. There is no definite $\mathrm{NH}_{2}$ emission in the central part of the coma; however, the $\lambda 6300$ line of $\mathrm{NH}_{2}$ may be hidden by the strong airglow line (the main lines of the airglow are strong on all spectrograms). The continuous spectrum is purely solar, as is revealed by the strength of $\mathrm{H}, \mathrm{K}$, and other absorption lines. The molecular emission in the comet is an extremely small fraction of the continuous emission.

Owing to difficulties in guiding, the diameter of the region of the coma showing a solar spectrum cannat be determined accurately. A rough estimate from spectrograms B371 and $\mathrm{B} 445$ gives a maximum value of $8000 \mathrm{~km}$. On the other hand, the diameter of the $C N$ emission, which extends to large distances from the nucleus in regions where no continuous background appears, may be estimated fairly accurately from B376 to be of the order of $315,000 \mathrm{~km}$. This may be compared with the diameters in Comet 19481, ${ }^{3}$

TABLE 1

COMET SPECTRA

\begin{tabular}{|c|c|c|c|c|c|c|c|c|c|c|}
\hline \multirow{2}{*}{$\begin{array}{c}\text { COMET } \\
\text { AND } \\
\text { FILM } \\
\text { No. }\end{array}$} & \multirow{2}{*}{$\begin{array}{c}\text { MAR., } \\
1951, \\
\text { DATE } \\
\text { (U.T.) }\end{array}$} & \multirow{2}{*}{$\begin{array}{l}\text { ExP. } \\
\text { (MIN.) }\end{array}$} & \multirow{2}{*}{ Emulsion } & \multirow{2}{*}{$\begin{array}{l}\text { MrD- } \\
\text { ALt. }\end{array}$} & \multicolumn{3}{|c|}{ SuIt } & \multirow{2}{*}{$\begin{array}{c}\text { REGION } \\
\lambda \lambda\end{array}$} & \multirow{2}{*}{$\begin{array}{c}r \\
\text { (A.U.) }\end{array}$} & \multirow{2}{*}{$\begin{array}{c}\text { RE- } \\
\text { MARKS }\end{array}$} \\
\hline & & & & & $\stackrel{w}{w}$ & $\left({ }^{\prime \prime}\right.$ Arc $)$ & p.a. & & & \\
\hline \multicolumn{11}{|l|}{ 1950b: } \\
\hline B306. & 1.42 & 60 & $103 a-\mathrm{F}^{*}$ & $27^{\circ}$ & 0.10 & 290 & $52^{\circ}$ & $3600-6700$ & 2.62 & \\
\hline & 10.38 & 70 & & 20 & .20 & 29 & 41. & $3600-6700$ & 2.64 & Clouds \\
\hline B376. & 11.43 & 95 & $103 a-\mathrm{F}^{*}$ & 22 & 20 & 290 & 29 & $3600-6700$ & 2.64 & Clouds \\
\hline B44 & 19.38 & 30 & $103 a-0$ & 23 & 20 & 290 & 20 & $3500-5000$ & 2.66 & Clouds \\
\hline $\begin{array}{l}\text { Qf/1 } \\
13108\end{array}$ & 20.28 & $(60)$ & $103 a-\mathrm{O}$ & 27 & .012 & 17 & 20 & $3200-4000$ & 2.66 & Clouds \\
\hline \multirow{2}{*}{\multicolumn{11}{|c|}{$\begin{array}{l}\text { 1951a: } \\
\text { B307. }\end{array}$}} \\
\hline & 1.49 & 45 & $103 a-\mathrm{F}$. & 13 & .10 & 290 & 174 & $3600-6500$ & 0.92 & \\
\hline B315. & 2.50 & 40 & $103 a-\mathrm{F}^{*}$ & 13 & 0.20 & 290 & 172 & $3600-6700$ & 0.93 & Clouds \\
\hline
\end{tabular}

* Ammonia-hypersensitized.

at $r=2.21$ : for $C N, 166,000 \mathrm{~km}$; for $\lambda 4050$, approximately $22,000 \mathrm{~km}$ (the continuous spectrum extending to somewhat smaller distances ${ }^{10}$ than $\lambda$ 4050).

This emphasizes once more the fact that the dimension of the coma has no meaning if it is not referred to definite spectral emissions. In practically all comets the solid particles reflecting the solar spectrum are present only in the central part of the coma, and each molecule has its specific extension. Whenever the $\mathrm{CH}, \mathrm{NH}_{2}$, and $\lambda 4050$ emissions appear, they are found approximately in the same central regions as the continuous spectrum, although the latter is still more confined to the regions close to the nucleus. A comet may therefore have a very large coma in the ultraviolet ( $C N$ emission) and an extremely small one in the visual region (continuous spectrum).

The present observations of Comet 1950b show that the evolution toward a pure continuous spectrum, as the comet recedes from the sun, continues beyond $r=2.21$ (Comet 1948l) to $r=2.66$. $C N$ declines in intensity relative to the solar spectrum, the $\mathrm{NH}_{2}$ and $\lambda 4050$ emissions practically disappear before $r=2.6$, and no trace remains of $\mathrm{C}_{2}, \mathrm{CH}, \mathrm{OH}$, and $\mathrm{NH}$. More extensive material is needed before we may definitely state that the increase of the intensity ratio $\lambda 4050 / C N$ with increasing heliocentric distance,

${ }^{10}$ Jose and Swings, op. cit., Fig. 1, facing p. 42, spectrum of February 2, 1949. 
which has been observed ${ }^{4}$ to $r=2.21$, ceases before $r=2.6 . C N$ may have been unusually abundant in Comet $1950 \mathrm{~b}$, around $r=2.6$.

Of course, a comparison between Comet $1948 \mathrm{l}$ at $r=2.21$ and Comet $1950 \mathrm{~b}$ at $r=$ 2.66 may not be fully justified: one object may be old and the other new. However, it appears safe to conclude that a comet at large heliocentric distance has a continuous spectrum, apparently due to the reflection of solar radiation by solid particles. It may be of interest to pursue this matter further, especially by determining colors. Parentmolecules, such as $\mathrm{CH}_{4}, \mathrm{NH}_{3}, \mathrm{CO}_{2}, \mathrm{~N}_{2}, \mathrm{O}_{2}$, and $\mathrm{CO}$, which are chemically stable and have no fluorescence spectrum in the observable region, may add their Rayleigh scattering to the reflection by solids. This could be checked by careful determination of the colors of a few distant comets.

\section{THE SPECTRUM OF COMET 1951a (PAJDUSAKOVA)}

Two spectrograms were obtained at heliocentric distances 0.92 and 0.93 . The airglow emissions are strong. While $C N$ is present, there is no evidence for $C_{2}$; there may be a trace of $\mathrm{NH}_{2}$ and of the $\lambda 4050$ emission. The main characteristic of the spectrograms is the strong continuous spectrum, confined to the central part of the coma; the contribution of the molecular emission to the brightness of the comet is extremely small compared with that of the continuous spectrum. The diameter of the $C N$ region is of the order of $46,000 \mathrm{~km}$, while that of the continuum is smaller than $6400 \mathrm{~km}$.

Comet 1951a appears unusual. To our knowledge, no other comet observed at a heliocentric distance less than 1 A.U. has ever shown such a strong continuum relative to the gaseous emission. On the basis of Oort's theory, this comet would be placed among the new ones, which, coming from the confines of the solar spectrum, have entered for the first time into the region near the sun. It is a striking example of a case where Oort's process 1 remains much more efficient than process 2, even at the small heliocentric distance $r=0.92$. It is hoped that photometric determinations and more extensive spectroscopic observations of this comet have been made at other observatories; also, that accurate positions are available for a determination of the original orbit.

On the basis of Baldet's compilation of observational data, Oort and Schmidt did not find any conspicuous difference between new and old comets with regard to intensity ratios of different emission bands. While our material on 1951a is not sufficient to make any definite statement, it seems that the intensity ratios $C_{2} / C N$ and $C H / C N$ are abnormally low for $r=0.92$. In this comet, probably a new one, $C_{2}$ and $C H$ would therefore be deficient relative to $C N$. This point will be kept in mind for our future observations. It may be that $1950 \mathrm{~b}$ is also a new comet and that the strength of $C N$ relative to $\lambda 4050$-compared to 19481 - is a result of the "freshness" of the comet. 